\title{
Suchtmedizinische Grundversorgung in der Allgemeinmedizin?
} Albrecht Ulmer

\author{
Basic Care for Substance-Related Disorders in General Medicine?
}

\section{Zusammenfassung}

Suchtmedizinische Grundversorgung muss differenzierte Behandlung einschließen. Angesichts der großen Zahl betroffener Patienten muss jeder Arzt dafür kompetent gemacht werden. Das bedeutet eine ganz andere Struktur, als wir sie heute haben. Wenn ein neuer Anlauf unternommen wird, alle Ärzte einzubeziehen, muss auch das mit neuen Strukturen unterlegt werden. Viel mehr Kontrolle der Struktur als des Einzelnen (!), Förderung statt Restriktion, Abbau aller überflüssigen Administration - so können Ärzte wieder für ihren unverzichtbaren Anteil an der therapeutischen Versorgung Suchtkranker zurückgewonnen werden, aus dem sie sich zurzeit so sehr zurückgezogen haben. Wir könnten ein Vielfaches der heutigen Effektivität erreichen und auch wieder der Freude an einem an sich faszinierenden, wunderbaren Fach.

\section{Schliuisselwörter}

Suchtmedizinische Grundversorgung · Allgemeinmedizin · ambulante Entzüge · Alkoholsubstitution · Struktur · Studium · Buprenorphin

\section{Abstract}

Primary care in addiction must include differentiated treatment. As a very great number of patients suffer from addiction, every physician should be able to deliver addiction treatment. This would require a completely different health care structure. A new attempt at including all physicians in addiction treatment would also require new structures. Control of structures rather than of individuals (!), support rather than restrictions, reduction of all superfluous administration - those are the measures that would entice physicians to assume again their indispensable part in the treatment of addicts, from which they tend to withdraw at present. We could multiply the efficacy of addiction treatment and rediscover the excitement of this fascinating field of medical treatment.

Key words

Basic care for addicted patients - general practioner $\cdot$ outclinic detoxification - maintenance therapy for alcoholics - structure . study $\cdot$ buprenorphine
Was heißt Grundversorgung? Haben wir uns an falsche Vorstellungen gewöhnt? Bei Diabetes, Hypertonie und Asthma z.B. heißt Grundversorgung:

Das Gros der Allgemeinärzte ist in der Lage, die Patienten differenziert einzustellen und zu versorgen.

Dies geschieht vor dem Hintergrund, dass es zusätzlich ein gut ausgebautes Spezialistennetz gibt, das vor allem für schwierige Fälle, aber auch generell zur Hebung der Qualität der Grundver- sorgung zur Verfügung steht. Hinzu kommt eine Festigung durch eine Fülle praxisrelevanter Forschung.

Suchtkrankheiten sind mindestens so häufig und eingreifend wie diese häufigen Krankheiten. Es muss also eine vergleichbare Zahl an Ärzten geben, die in der Lage sind, eine differenzierte Einstellung und Versorgung zu gewährleisten. Das muss Grundversorgung heißen. Wenn mit Grundversorgung etwas anderes gemeint ist, z. B. nur das Diagnostizieren von Suchtkrankheiten und die Weiterleitung an Spezialzentren, geht das noch von der 
falschen Prämisse aus, dass Suchtkranke überwiegend keiner medikamentösen und damit ärztlichen Behandlung bedürften. Noch heute ist der Glaube daran auch in der professionellen Suchthilfe weit verbreitet und die nötigen Kapazitäten stehen infolge dieser Sicht bei weitem nicht bereit.

Die heutige Situation ist von katastrophal schlechten Erreichbarkeitsquoten und damit nicht akzeptablen Misserfolgszahlen geprägt. Es ist bekannt, dass die meisten Alkoholkranken Hausärzte aufsuchen, aber dort auf kein qualifiziertes System stoßen. Will man diese Hausärzte nun darin schulen, bei ihren Patienten Suchtkrankheiten zu beachten und anzusprechen, etwa im Sinne des Motivational Interviewing, ist das eine nachgewiesen gute Idee. Aber eine Grundversorgung ist das noch nicht. Auch jetzt schon werden Suchtkranke in Arztpraxen viel auf das qualifizierte Suchthilfeangebot hingewiesen, aber jeder Hausarzt kann ein Lied davon singen, wie oft dieser Hinweis ins Leere geht.

Das liegt weniger an mangelnden Ausbildungen im Motivational Interviewing als an der im Suchthilfesystem verankerten Vorstellung, dass das eigentlich qualifizierte Hilfesystem erst hinter dem Hausarzt oder „oberhalb“ von diesem beginnt und dass es nur relativ weniger ärztlicher Suchtkrankenbehandlung bedürfe. Dazu passt auch die derzeitige Vorstellung von suchtmedizinischer Qualifikation, die so eingerichtet wurde, dass sie de facto nur von sehr wenigen, ausgesuchten Ärzten erworben und von noch weniger praktisch angewendet wird.

Wir müssen aufwachen! Wir obliegen einer Suchtkranken in großer Zahl nicht gerecht werdenden Vorstellung und haben die Suchtkrankenbetreuung in ganz falsche Strukturen gedrängt.

Suchtkranke müssen bei der Mehrzahl ihrer Arztbesuche auf einen Ansprechpartner treffen, der ihre Situation kompetent einstufen und sie aus eigener Erfahrung differenziert über die Behandlungsmöglichkeiten beraten kann, auch und besonders über die medikamentösen. Um das zu erreichen, muss zumindest jeder Hausarzt, ja eigentlich jeder Arzt dafür differenziert ausgebildet sein. Suchtmedizinische Qualifikation muss also fester Bestandteil der Ausbildung zum Arzt werden.

\section{Suchtmedizinische Qualifikation gehört ins Medizinstudium.}

Ergänzt durch reflektierende Sitzungen, zumindest in den ersten Berufsjahren, lässt sich so allmählich erreichen, dass Suchtkranke bei der überwiegenden Zahl von Ärzten - und nicht erst irgendwo weitergeschickt, wo die meisten beim bisherigen System nie ankommen - und dort auf ein qualifiziertes Hilfesystem treffen, wo unmittelbar eine differenzierte Behandlung eingeleitet wird.

Die Allgemeinmedizin hat diese Notwendigkeit schon lange gespürt und mehr als alle anderen medizinischen Fachsparten zu reagieren versucht. Sie hat darin - gezwungenermaßen - sogar eine Führungsrolle übernommen. Unter anderem ist daraus die Dt. Gesellschaft für Suchtmedizin entstanden. Ihr Vorsitzender ist ein Allgemeinmediziner, seine Vorgänger - einer davon ich waren es auch. Allgemeinmediziner haben die ersten zehn Jahre der Gesellschaft entscheidend mitgeprägt.
Die Allgemeinmedizin ist nicht nur „allgemein“ = unspezialisiert, sondern auf einige Dinge, die der Suchtkrankenbetreuung besonders zugute kommen, besonders spezialisiert, z. B.

- $\quad$ auf Querverbindungen und

- aufzufangen, was sonstige Spezialisten vernachlässigt oder schon aufgegeben haben.

Dazu kommt das Spezifikum, das von der umfassenden Zuständigkeit vom Fußpilz über Psychose bis zum Mammakarzinom, von Wohnortnähe und der Betreuung ganzer Familien mit mehreren Generationen geprägt wird. Psychologisch gesehen prägt das ein systemisches Denken und medizinisch gesehen ganzheitliche Ansätze. Beides kommt Suchtkranken besonders zugute.

$\mathrm{Zu}$ erwähnen ist auch, dass es unter Allgemeinärzten einen hohen Anteil an Idealismus gibt, der zwar im Sinne eines Helfersyndroms gefährlich ist, den aber Suchtkranke oft brauchen, damit krankheitsbedingte Sperren überwunden werden. Allgemeinärzte leisten hier viel, was niemand richtig sieht und anerkennt. In den 90er-Jahren hat die Allgemeinmedizin eine hohe Bereitschaft gezeigt, eine flächendeckende Grundversorgung zu gewährleisten. Die Gesamtleistung der Allgemeinmedizin gegenüber Suchtkranken ist insgesamt großartig.

Aber: Wenn die Allgemeinmedizin sich ein Feld wie die Suchtmedizin überwiegend aus sich selbst heraus erarbeitet, dann hat das auch seine Probleme. Es geschieht mit einer gewissen Hemdsärmeligkeit, auf der Basis des Erfahrung-Weitergebens von Arzt zu Arzt, mit unzureichender Standardisierung und Qualitätskontrolle.

Am krassesten wurde das in den 90er-Jahren an der Codeinsubstitution manifest, vor allem in Bayern, wo zwar sehr viel Gutes geleistet wurde und die Todeszahlen nach oben schnellten, als es verboten wurde. Aber quasi verboten wurde es, weil sich immer mehr zeigte, wie viel nicht zu verantwortende Schlamperei sich eingeschlichen hatte, wo weder die Bereitschaft der Ärzte reichte, sich gründlich kompetent zu machen, noch konkret am Patienten mit gewissenhafter Gründlichkeit zu arbeiten.

Die Folge war, dass sich die Ansicht durchsetzte: Der Allgemeinmedizin kann man das nicht überlassen, zumindest nicht alleine. Die Reaktionen waren in erster Linie restriktiv, mit den Mitteln des Betäubungsmittelgesetzes, in zweiter Linie auch strukturierend mit neuen Ansätzen eines Qualitätsmanagements: der Einführung einer Fachkunde und von Qualitätssicherungskommissionen.

Die Folge davon ist jetzt wieder eine Sackgasse: Mehr denn je sind wir von der Idee einer Grundversorgung entfernt. Zwar ist manche gröbste Schlamperei ausgemerzt, dafür aber fehlen tausende Ärzte als kompetente Ansprechpartner und es fehlen - von einzelnen regionalen Bemühungen abgesehen - Zeichen des Aufbaus und der Förderung. Stattdessen leidet das existierende Rumpfsystem unter einem Gefühl des Misstrauens (BtMG, Kommissionen) und unsäglicher, völlig überflüssiger administrativer Überlastung. Auf der Webseite der DGS steht der Hinweis, dass ein Arzt 10000 Euro Strafe zahlen muss, weil er fünf statt nur drei stabile Substitutionspatienten weiterbehandelt hat. Und innerhalb des letzten Monats haben sich gleich drei Kollegen bei 
mir gemeldet, gegen die wegen ihres suchtmedizinischen Engagements staatsanwaltlich ermittelt wird. Nachrichten dieser Art reißen nicht ab. Sie haben eine verheerend abschreckende Wirkung auf die Ärzte: Lasst euch bloß nicht auf dieses verminte Gebiet ein! Weithin Überregulierung und schlechte Stimmung. So gelangen wir nicht zu einer Vision von etwas Gutem.

Nun hatten die drei Kollegen tatsächlich, wie man sagt, „Mist gebaut". Ein Todesfall beispielsweise am dritten Tag nach Beginn einer Methadonsubstitution bei einer inzwischen erreichten Tagesdosis von $140 \mathrm{mg}$ zeigt ein grobes und wieder lebensgefährliches Abweichen vom fachlich notwendigen Standard. Es fehlte obendrein ein Ausgangsurinbefund. Offensichtlich gelingt es mit den derzeitigen restriktiven und administrativen Mitteln nicht, solche Vorkommnisse zu verhindern. Es gelingt, die Vorkommnisse deutlich zu ahnden, so dass das System noch restriktiver und ausgedünnter wird und damit nicht besser, sondern viel schlechter.

Zwei dieser Kollegen, die sich bei mir gemeldet haben, kenne ich seit Jahren als Ärzte, die sich besonders viele Gedanken um eine suchtmedizinische Versorgung ihrer Region gemacht haben, die deshalb schon lange mit der damaligen DGDS Verbindung aufgenommen hatten. In einem Fall weiß ich auch um die zutiefst ärztliche, ethisch hervorragende Grundhaltung des Kollegen.

Warum passieren solchen Ärzten solche fachlichen Fehlleistungen? Offensichtlich, weil es an einer passenden, gesunden Struktur um ihre Arbeit herum fehlt. Es fehlt die Einbettung in Konzepte, an denen die universitäre Forschung, Kliniken und viele andere Professionen stützend beteiligt sind. Mehrheitlich sind ärztliche Einzelkämpfer betroffen. Oft entsteht der Eindruck einer sehr ungesunden Überlastung in einer Umgebung, die sie viel zu allein gelassen hat. Sie haben ihren Kopf hingehalten und „wer etwas tut, macht auch Fehler“. Für Fehler muss man gerade stehen, aber wenn das dazu führt, dass es die Engagiertesten und trotz solcher Fehler immer noch einige der Besten „heraushaut“, dann stimmt die Struktur nicht, und mit aller Verfolgung und Bekämpfung solcher Überengagements wird sie nur noch schlechter.

Im Dezember 2002 fand in Avignon auf Initiative von Robert Newman ein fünftägiger Workshop mit etwa 25 Experten aus 13 Ländern über die Erfahrungen mit Substitutionsbehandlungen vor allem bei Allgemeinärzten unter verschiedensten Bedingungen statt. Heft 4 dieses Jahres hat bereits einen Bericht darüber enthalten. Die Teilnehmer kamen aus Kanada, USA, Schottland, England, Holland, dem gastgebenden Frankreich, Deutschland, Österreich, Schweiz, Italien, Kroatien, Iran und Australien. Deutschland war durch die Apothekerin Christiane FahrmbacherLutz, die zu Recht eine mögliche tragende Rolle engagierter ApothekerInnen für gute regionale Strukturen hervorhob, und mich vertreten.

Zentrale Fragen des Workshops brachte der australische Kollege Nicholas Litzeris auf den Punkt:

Was wollen wir (die Gesellschaft, das Gesundheitssystem) für die Behandlung Abhängiger von unseren Allgemeinärzten?

- Welchen Anteil von ihnen möchten wir involviert haben?
- Welche Aufgaben sollen sie erfüllen?

- Auf welche Weise erreichen wir das am besten?

- Mit welchen Mechanismen lässt sich das am besten kontrollieren?

Er berichtete, dass in seinem Land 50\% der Allgemeinärzte ein bis zwei Substitutionspatienten betreuen, während sich 1-5\% als speziell interessiert gezeigt haben und größere Zahlen bis zu 200 Patienten betreuen. Umgekehrt ging er auch vom unterschiedlichen Hilfebedarf der Patienten aus. Nur etwa $10-20 \%$, so seine Schätzung, benötigen eine langjährige Betreuung in spezialisierten Einheiten, während die überwiegende Mehrheit teils viel besser in einer allgemeinärztlich integrierten Behandlung zu betreuen ist.

Eine ähnliche Erfahrung wurde aus vielen Ländern berichtet. Das Thema Zentrierung der Patienten an relativ wenigen Zentren versus dezentraler Betreuung bei Allgemeinärzten erwies sich als zentrales Thema. Es hat viel zu tun mit dem Erscheinungsbild dieses Behandlungsangebots, worin sich seine Akzeptanz und Effektivität widerspiegeln. Auch in Deutschland spielt das eine erhebliche Rolle, leisten wir uns doch in einer Stadt wie Stuttgart eine Substitutionsstruktur mit 10-15 Ärzten, während beispielsweise Basel mit halb so vielen Einwohnern die Substitution auf 250 Ärzte verteilt hat.

Die Beiträge aus allen Ländern zeigen: Eine adäquate (= optimale), flächendeckende Betreuung der opiatabhängigen Patienten ist allein auf der Basis spezialisierter Zentren nirgends möglich. Überall braucht es daneben eine viel integriertere, dezentrale Betreuungsstruktur, die nur unter breitem Einbezug der Allgemeinärzte zu realisieren ist.

Eines der interessantesten Modelle, vielleicht das interessanteste, bietet seit wenigen Jahren Frankreich. Praktisch ohne Restriktionen wurde es allen Ärzten erlaubt, Opiatabhängige mit Buprenorphin zu behandeln, womit innerhalb weniger Jahre eine Behandlungsintegration von weit mehr Patienten als in allen vergleichbaren Ländern, eine Reduktion der Drogentodeszahlen um $80 \%$ und eine ähnlich drastische Reduktion der Kriminalität erreicht werden konnten. William Lowenstein, Paris, und Marc Auriacombe, Bordeaux, stellten diese Effekte in Kontrast zu einem hochschwelligen, restriktiven Methadonangebot, mit dem gerade mal 10\% der Behandelten erreicht wurden und das praktisch keinen Effekt auf die Gesamtentwicklung hat. Buprenorphin als „leichteres“ Opiat bietet mit einer einzigartigen Intoxikationssicherheit eine breite Möglichkeit, den Umgang mit Substitutionsmitteln weit gehend den Betroffenen anzuvertrauen. Die Effekte in Frankreich sprechen für sich und sollten auch bei uns noch mehr zu denken geben. Angesichts der strukturellen Notlage führt eine sorgfältige Abwägung klar zu folgender Forderung:

Buprenorphin ist aus den betäubungsmittelrechtlichen Regelungen herauszunehmen und Opiatabhängigen in großer Breite zur Verfügung zu stellen.

Newman fasste so zusammen: Mit der notwendigen Unterstützung und dem Zutrauen in niedergelassene Ärzte kann die Erreichbarkeit der Behandlung schnell und erheblich erweitert 
werden - durchaus in Verbindung mit Angeboten an spezialisierten Einheiten. Wo das wirklich gewollt und begleitet wird, ist eine erhebliche Erweiterung auch da gut möglich, wo es an Finanzen, personellen Ressourcen, Spezialisierungsmöglichkeiten und beispielsweise psychosozialen Begleitangeboten mangelt.

Erwähnenswert ist auch die von den kroatischen Kollegen Ivancic und Tiljak vorgestellte Struktur, die Substitutionspatienten praktisch Hausärzten zuteilt, mit dem Effekt, dass meist nur ein bis drei Substitutionspatienten pro Arzt behandelt werden und nirgends Substitutionsszenen entstehen.

Mit allen, oft gut gemeinten Behandlungsrichtlinien muss sich jede Gesellschaft fragen, wie weit diese Richtlinien zu einer Verknappung und Zentrierung des Behandlungsangebots führen, und jede Gesellschaft muss sich fragen, wie viele Substitutionsszenen (= Desintegration) sie sich leisten will.

Was heißt das alles für die Vision einer guten suchtmedizinischen Grundversorgung in der Allgemeinmedizin?

1. Heraus aus der Sackgasse, in der es immer noch weniger Ärzte werden, die zu einer suchtmedizinischen Grundversorgung bereit sind.

2. Dafür Sorge tragen, dass so schnell wie möglich jeder Arzt ein ausreichendes suchtmedizinisches Basiswissen besitzt, um Suchtkranke selbst differenziert zu behandeln.

3. Einbinden dieses neuen Ansatzes in gute, stützende Strukturen, die der Arzt als Förderung seiner Arbeit erleben kann:

a) ein von der Politik gefördertes und überwachtes, lebendiges Miteinander von Forschung, Praxis und Lehre bisher flächendeckend kaum im Ansatz zu erkennen;

b) interdisziplinäre Netzwerke bis hin zu Polizei, Justiz und Politik;

c) weitere Unterstützung, soweit sich konkreter Bedarf zeigt;

d) motivierende Honorierung guter Arbeit.

4. Bei Kontrollen viel mehr die Systeme kontrollieren als alle Einzelnen. Wenn Einzelne versagen, ist das oft ein Hinweis auf Fehler im System. Kontrollen so, dass sie wieder als Förderung erlebt werden.

5. Solange viele Ärzte eine Art Notverwaltung einer fehlentwickelten Struktur leisten müssen, brauchen sie Zeichen des Verständnisses und der Anerkennung. Vertrauen kann man nicht einfordern, wo es nicht da ist. Aber es ist notwendig für gute Arbeit. Vertrauen ist eine Strukturfrage.
Zur Grundversorgung gehören:

- das differenzierte Erkennen von Suchtkrankheiten, mit einem geschulten Blick für zugrunde liegende Strukturen, etwa im familiären oder sozialen Milieu oder im Betroffenen selbst;

- das kompetente, differenzierte Ansprechen der Betroffenen, wobei für diese ersichtlich sein muss, dass der Arzt konkrete, auch eigene Hilfsangebote bereit hält, und sei es zunächst nur: „Ich biete Ihnen an, dass wir uns darüber noch mal mehr unterhalten“;

- ein eingeführter Kontakt zu den suchttherapeutischen Einrichtungen, mindestens ähnlich so eingeführt wie der Kontakt zu den umgebenden Kliniken, darüber Einbezogenheit in strukturierte Konzepte der Region;

- ein differenziertes, medikamentöses Angebot für betroffene Patienten: Wo sich der Arzt nicht kompetent genug fühlt, müssen ihm eine kompetentere Praxis und/oder ein entsprechendes Zentrum zur Seite stehen;

- Bereithalten der wichtigsten substituierenden Behandlungsmöglichkeiten durch eine viel größere Zahl von Ärzten. Diese werden es tun, wenn sie sehen, dass die Bedingungen stimmen;

- Bereithalten standardisiert gut strukturierter, ambulanter Entzugsangebote für Alkoholabhängige;

- Bereithalten eines differenzierten, medikamentösen Behandlungsangebots für Patienten mit Alkoholproblemen für die Zeit nach dem Entzug;

- substituierende Behandlungsansätze für Alkoholabhängige müssen in guter Kooperation zwischen Universität und Praxen entwickelt werden - unbedingt!

- Kompetenz in Raucherentwöhnungsberatung und -behandlung;

- und immer wieder: eine gute Struktur, die das alles trägt. 